\section{Depression}

Auf einem Roundtable des Unternehmens Aristo Pharma zur Anwendung von Tranylcypromin (Jatrosom ${ }^{\circledR}$ ) hat sich gezeigt, dass niedergelassene Psychiater oft wenig Erfahrung mit dem Monoaminoxidase-Inhibitor haben. Dr. Patrick Thilmann, niedergelassener Psychiater in Mannheim, hatte vor seiner Zeit als Niedergelassener ebenfalls keine Berührungspunkte mit dem Antidepressivum. In den letzten sieben Jahren habe er aber gute Erfahrungen gesammelt und von den Patienten immer wieder einen schnellen Wirkeintritt berichtet bekommen. Er habe nur zwei Patienten mit Nebenwirkungen gehabt, so Thilmann. Beide Fälle seien allerdings vermeidbar gewesen, denn die Patienten hätten offensichtlich und eigenmächtig gegen die vereinbarten Behandlungsrichtlinien verstoßen. Zweimal sei die tyraminarme Diät nicht eingehalten, einmal sei gleichzeitig eine zu hohe Dosis eingenommen worden. Tranylcypromin ist zur Therapie von Patienten mit depressiven Störungen, unabhängig ihrer nosologischen Einordnung (Jatrosom ${ }^{\circledast} 10 \mathrm{mg}$ ) sowie für Patienten mit Major-Depression, wenn mindestens zwei Standardantidepressiva (einschließlich eines Trizyklikums) wirkungslos oder unverträglich waren (Jatrosom $20 \mathrm{mg}$ ), zugelassen.

Aristo Pharma

\section{Neu auf dem Markt}

Das Unternehmen neuraxpharm erweitert erneut sein Antidementivaportfolio. $\mathrm{Ab}$ sofort ist neben den Cholinesterasehemmern Donepezil-neuraxpharm ${ }^{\circledast}$ und Rivastigmin-neuraxpharm ${ }^{\infty}$ auch der NMDA-Rezeptorantagonist Memantinhydrochlorid erhältlich. Memantinhydrochlorid-neuraxpharm ${ }^{\circledast}$ ist zur Behandlung der moderaten bis schweren Alzheimer-Demenz zugelassen und gilt als leitliniengerechte Therapie. Memantinhydrochlorid-neuraxpharm ${ }^{\varpi}$ ist in den Wirkstärken $5 \mathrm{mg}, 10 \mathrm{mg}, 15 \mathrm{mg}$ und 20 mg als Filmtabletten sowie als Lösung mit $10 \mathrm{mg} / \mathrm{ml}$ erhältlich. Die Filmtabletten der höheren Wirkstärken sind teilbar (15 mg: drittelbar, 20 mg: viertelbar).

neuraxpharm

Bipolar-I-Störungen

\title{
Manische und depressive Symptome bei gemischten Episoden verbessern
}

Bipolare Störungen sind ein komplexes Krankheitsbild, das sich neben den charakteristischen Stimmungsschwankungen auch durch Störungen der kognitiven Leistungsfähigkeit und der Funktionalität auszeichnet. Gerade die Beeinträchtigungen der Alltagsfunktionen seien mit erheblichen medizinischen, sozialen und gesundheitsökonomischen Belastungen verbunden, berichtete Professor Ana González-Pinto, Vitoria/Spanien. Das Auftreten depressiver Symptome in manischen und hypomanischen Phasen könne die Kognition und die Funktionalität der Patienten noch zusätzlich beeinträchtigen und das Management der Erkrankung erschweren. Das Erreichen einer klinischen Remission (definiert als sechsmonatige Symptomfreiheit) reiche nicht aus, so González-Pinto. Wichtig sei darüber hinaus die Wiederherstellung der beruflichen und sozialen Funktionalität. Unter diesem Aspekt sei es wichtig, spezifische Symptomkonstellationen bei den einzelnen Patienten zu identifizieren und entsprechend zu therapieren.

Mit Asenapin könne sowohl in Monotherapie als auch in Kombinationstherapie mit Stimmungsstabilisierern eine schnelle und anhaltende Reduktion manischer Symptome erreicht werden, berichtete Professor Roger McIntyre, Toronto/Kanada. Schon am zweiten Behandlungstag sei in den klinischen Studien eine gegenüber Placebo signifikante Verbesserung der Manie beobachtet worden. „Eine frühe Verbesserung ist ein robuster Prädiktor einer späteren Response
Besserung depressiver Symptome

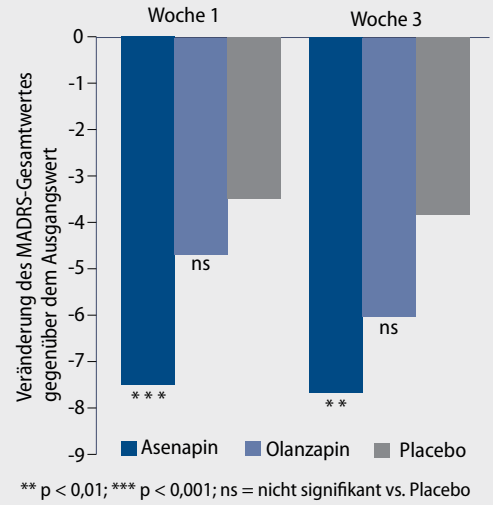

1 Veränderung des MADRS (MontgomeryÅsberg Depression Rating Scale)-Gesamtwertes nach einer und drei Wochen gegenüber Baseline [nach: Azorin JM et al., ECNP Wien 2012, Poster P.2.3.019]

und Remission“, betonte der Psychiater. Die Ergebnisse einer Post-hoc-Analyse bei Patienten mit gemischten Episoden zeigen, dass Asenapin neben den manischen auch die depressiven Symptome signifikant im Vergleich zu Placebo verbessert ( Abb. 1). Olanzapin hatte dagegen keinen signifikanten Effekt auf depressive Symptome.

Abdol A. Ameri, freier Medizinjournalist

Symposium „Bipolar I disorder - Improving clinical and functional outcomes" 25. ECNP-Kongress, Wien, 14.10.2012 Veranstalter: Lundbeck

\section{Wahl der Vergleichstherapie bei der Bewertung von MS-Therapeutikum kritisiert}

Im Rahmen der frühen Nutzenbewertung nach dem Arzneimittelmarktneuordnungsgesetz (AMNOG) kommt das Institut für Qualität und Wirtschaftlichkeit im Gesundheitswesen (IQWiG) für den Wirkstoff Fampridin (Fampyra ${ }^{\circledR}$ ) zu dem Schluss, dass ein Zusatznutzen nicht belegt sei. Als zweckmäßige Vergleichstherapie hatte der Gemeinsame Bundesausschuss (G-BA) Physiotherapie gemäß Heilmittelrichtlinien festgelegt.

„Beim Vergleich einer medikamentösen mit einer nicht-medikamentösen Therapie nach den Methoden des IQWiG erge- 